Z. Rashid, B. Morova, Ö. Yaman, S. Soydan, Ö. Birer, I. Yilgor, and A. Kiraz*

\title{
Reconfigurable and Permanent Wetting Patterns on Polymer Surfaces Obtained Using Plasma Oxidation and Laser Ablation
}

https://doi.org/10.1515/odps-2018-0004

Received September 29, 2018; accepted October 25, 2018

\begin{abstract}
Smart surfaces with preferably reconfigurable wetting properties can lead to key applications in labon-a-chip analytical and preparative systems. In this paper, we present our recent results obtained using polymer surfaces whose wetting properties are modified in a permanent manner using laser ablation and in a reconfigurable manner using plasma oxidation. Polydimethylsiloxane (PDMS) diluted in solvent is used as the polymeric material coated over microscope glass slides in our studies. In the first part, the tracks of $\sim 70 \mu \mathrm{m}$ width are defined over the surface by surface oxidation using cold plasma exposure through a microfluidic channel. In the second part, femtosecond laser micromachining is used for selective removal of polymer coating and uncovering the hydrophilic glass substrate. We experimentally demonstrate guiding of water in the form of filaments and droplets over the obtained hydrophilic tracks of $\sim 110 \mu \mathrm{m}$. We also discuss preliminary experiments to coat light sensitive azobenzene over a glass substrate with the help of a silane in order to achieve reversible isomerization upon periodic exposure to $U V / v i s$ radiation. Furthermore, we elaborate advantages, challenges and the significant role of such patterned surfaces in future applications.
\end{abstract}

Keywords: Superhydrophobic, hydrophilic, isomerization, laser ablation, recovery, oxidation

Z. Rashid: Department of Electrical and Electronics Engineering, Koç University, Sariyer, 34450 Istanbul, Turkey

B. Morova: Department of Physics, Koç University, Sariyer, 34450 Istanbul, Turkey

Ö. Yaman: Department of Physics, Koç University, Sariyer, 34450 Istanbul, Turkey

S. Soydan: Department of Physics, Istanbul Technical University, Maslak, 34469 Istanbul, Turkey

Ö. Birer: ASELSAN Radar and Electronic Warfare Systems Business Sector, Golbasi, 06830 Ankara, Turkey

I. Yilgor: Department of Chemistry, Koç University, Sariyer, 34450 Istanbul, Turkey

\section{Introduction}

Chemical surfaces with adjustable wettability from hydrophobic to hydrophilic state have important uses in the fields of medicine, security, microchemistry and industrial microbiology [1]. Superhydrophobic surfaces, due to their water repellent nature, provide various key properties such as drag reduction [2], corrosion resistance [3], anti-fogging [4] and anti-icing [5] over power transmission lines and aircraft wings to drastically increase their life time. The aqueous droplet supported by a superhydrophobic surface acquires a spherical shape which serves as an optical microcavity for the amplification of stimulated emission forming a laser with biological gain medium [6]. The extent of hydrophobicity is evaluated by putting a drop of water (normally $10 \mu \mathrm{L}$ ) over the substrate and measuring the angle called contact angle (CA) between the droplet and the substrate surfaces at the point of their contact. Superhydrophilic surfaces, on the other hand, allow water to spread isotropically in a quick span of time resulting extremely low CA $\left(<5^{\circ}\right)$, thus, they are water attractive in nature. Superhydrophilic surfaces are useful for self-cleaning [7], ultrafast drying [8] and heat transfer [9].

For the last two decades, numerous studies have shown the applicability of hydrophilic surfaces in biosensing applications for providing an adhesive support to the reagents. Guiding or trapping the droplets over chemically heterogeneous and oil impregnated surfaces is a major scientific challenge having broad environmental impact on the modern society. The dynamic behavior of emulsion droplets over solid substrate is a function of many parameters such as degree of roughness, interfacial properties of droplet/surrounding liquid and ambient conditions which uniquely determine the operating regime of the fluidic system [10]. The interpretation of such liquid kinetics allows enzymes, genes, DNA and cells either separately or

\footnotetext{
${ }^{\star}$ Corresponding Author: A. Kiraz: Departments of Physics, Electrical and Electronics Engineering, Koç University, Sariyer, 34450 Istanbul, Turkey, E-mail: akiraz@ku.edu.tr
} 
even encapsulated in microdroplets to attach or trap efficiently over a functionalized region for long term analysis, incubation or further chemical/optical treatment [11-13]. The degree of enzyme or cell immobilization is an important parameter for determining the sensing efficiency especially when the sensing is carried out by optical means [14]. In this regard, the wettability of the underlying substrate should be tunable reliably by external mechanism to accordingly execute the enzymatic activity or diagnostic protocol in the microchannel [15].

Surfaces with a wide range of chemical compositions have been prepared which allow substantial change of their wetting behavior upon exposure to certain external stimuli such as light [16], electric field [17], magnetic field [18], solvents [19], plasma [20] and corona [21]. Among these, non-contact processes are of paramount importance because they offer remote control, contaminationfree, low temperature and precise exposure with high resolution [22, 23]. Surface modification with external noncontact stimulant mostly causes certain chemical activity such as isomerization, segmental migration or surface oxidation which enhances their bond polarity or surface reactivity. Photoisomerization of light sensitive materials upon exposure to $U V / v i s$ radiation for a certain period of time is a well-known method to achieve surface modification. In this regard, azobenzene and some of its derivatives are known to exhibit large changes in configurational geometry and dipole moments as a result of $U V / v i s$ irradiation. Trans state of azobenzene is hydrophobic and more stable state, whereas cis state is hydrophilic, which is achieved after $U V$ irradiation for a certain time. Recent advances in surface chemistry together with the versatility of organic reactions, made it possible to exploit azobenzene to achieve large changes in wetting properties, fast response and more cycles of wetting periodicity [24-26].

It is also possible to exploit the intrinsic properties of the most commonly used polymers in a simple way for achieving tunable wetting. Polydimethylsiloxane (PDMS) elastomer is a well-known and widely used soft polymer due to its durability, non-toxicity, quick cross linking, easy handling and, in addition, its ability to allow artificial roughness at the nanometer scale. Inherently, PDMS is a hydrophobic polymer with a water CA of $108^{\circ}-110^{\circ}$ which can be increased drastically up to $170^{\circ}$ by the addition of silica $\left(\mathrm{SiO}_{2}\right)$ nanoparticles or by laser micromachining $[27,28]$. The hydrophobicity of PDMS can be reduced chemically, by oxidizing its surface probe layer. Surface oxidation of PDMS coating can be achieved in a variety of ways; by plasma [29], UV/ozone [28] or corona discharge [21]. The fundamental principle in all these processes is the decomposition of atmospheric oxygen molecule to atomic oxygen which recombines with oxygen molecule to produce ozone. As a result of PDMS exposure to plasma or ozone, hydrophobic methyl groups are transformed into hydroxyl groups which are hydrophilic in nature [21]. Extended oxidation also produces a silica layer on top of PDMS. Upon mechanical or thermal stress, top brittle silica layer suffers crackdown causing low molecular weight (LMW) moieties from the bulk to migrate to the top to recover the hydrophobicity [30].

Controlling the motion of various polar liquids on a submicron scale over narrow hydrophilic tracks has produced novel applications in broad spectrum of droplet and surface microfluidics. Due to high surface to volume ratio, microfluidic systems are capable to perform micro reactions with a fast response time and reduced reagents consumption resulting high throughput sensing, screening and synthesizing assays. The transport of liquid along the hydrophilic pattern over a free surface takes place due to the surface tension pumping mechanism from one side called input reservoir to the other side called output reservoir [31, 32]. These systems are finding applications in surface microfluidics for the detection of explosives for homeland security and public safety [33]. Due to the presence of hydrophilic tracks, water follows the pattern unless equilibrium state is reached and pressure in both reservoirs is balanced. With PDMS used as substrate along with oxidation mechanism, it is quite simple to define hydrophilic channels which are erasable and rewritable due to the absolute recovery.

Another method of defining hydrophilic tracks over hydrophobic PDMS is exposing the surface with high power femtosecond $(f s)$ laser pulses which removes or ablates PDMS coating. In this way, the substrate surface which is hydrophilic glass is uncovered on a sub-micron scale. Such extremely narrow and selective removal of PDMS makes shallow tracks which are although irreversible and not too hydrophilic, yet, able to provide suffcient hydrophilicity to manipulate microdroplets flowing with the host fluid. This method physically modifies the surface and is irreversible in nature, ensuring permanent surface activation without any fear of hydrophobic recovery $[34,35]$.

In this manuscript, we demonstrate hydrophilization in a patterned way over PDMS elastomer to obtain guiding of water as a potential application. In the first part, PDMS coated surface is exposed to atmospheric pressure dielectric barrier microplasma as a surface modification technique to obtain hydrophilic regions on the surface. Since, cold plasma can enter microchannels and activate the surface, oil-in-water $(\mathrm{O} / \mathrm{W})$ and water-in-oil-in-water (W/O/W) droplet emulsions in microchip channels have 
been demonstrated recently by this technique [36]. Energetic photons, electrons or ions found in plasma leads to the homolytic cleavage of the $\mathrm{Si}-\mathrm{C}$ bonds present in the PDMS as pendant groups. Silicon and oxygen radicals recombine and create a molecularly thin silica layer on the surface. Formation of the silica layer results in a temporary or transient decrease in the hydrophobicity of the surface [37]. To achieve such narrow patterns on the PDMS surface, we used a microfluidic chip as a mask firmly placed on top of the PDMS-coated surface and with two holes punched at the two ends. As a result, exposed region becomes hydrophilic and when water is dropped on one side, it is driven along the channel due to the surface tension towards the other side. A thickness of as small as $\sim 70 \mu \mathrm{m}$ of the hydrophilic channel has been achieved with this method. In the second part, we obtain hydrophilic tracks by removing PDMS coating using laser ablation along inclined tracks which exposes the hydrophilic glass substrate surrounded by hydrophobic PDMS. We obtain guiding and sorting of microdroplets over these inclined tracks in a closed microfluidic environment. Third part deals with photoisomerization of azobenzene from trans to cis state upon $U V$ exposure and back to trans state after vis irradiation. We analyze absorption spectrum of the azobenzene coating over a glass slide to evaluate its isomeric state.

\section{Materials and Methods}

In order to fabricate hydrophobic polymer surfaces, we used PDMS elastomer (Dow Corning, Sylgard 184) coating over a glass slide for achieving hydrophilic tracks for surface microfluidics as well as droplet microfluidics demonstration. PDMS base agent was mixed with curing agent at 1:10 weight ratio and was kept in a desiccator for $20 \mathrm{~min}$ to remove bubbles. PDMS mixture was then diluted by mixing it with toluene at a weight ratio of (1:4). Glass slides (dimensions: $76 \mathrm{~mm} \times 26 \mathrm{~mm} \times 1 \mathrm{~mm}$ ) were cleaned by initially dipping in Hellmanex II (Hellma GmbH \& Co.) with $2 \%$ concentration in distilled water for $70 \mathrm{~min}$. Hellmanex II is an alkaline liquid detergent used for effective cleaning of quartz and glass slides. Subsequently, the glass slides were dried with pressurized nitrogen and then dipped in acetone, isopropyl alcohol (IPA) and distilled water for $30 \mathrm{~min}$ each. The diluted solution of PDMS was spin-coated over a clean glass slide at a speed of $6000 \mathrm{rpm}$ for $2 \mathrm{~min}$. Coated glass slides were kept in a $100^{\circ} \mathrm{C}$ oven for $30 \mathrm{~min}$ to cure the PDMS. This process produced a PDMS film with a thickness of around $1 \mu \mathrm{m}$ on the glass slides.
For the top layer, we fabricated a mold by $U V$ lithography of a $40 \mu \mathrm{m}$ thick coating of SU-8 2050 negative photoresist over a silicon substrate. The exposed sample was then developed to obtain features with widths varying from $50-800 \mu \mathrm{m}$. The mold was kept in closed environment with silane, hexamethyldisilazane (HMDS) for 12 hr so that HMDS vapors can coat well over the mold surface to make it hydrophobic, a process so called silanization. We obtained top PDMS layer using conventional soft lithography technique by pouring PDMS base agent mixed with curing agent over the mold and keeping in oven for $30 \mathrm{~min}$ at $100^{\circ} \mathrm{C}$. The solidified PDMS was cut and peeledoff from the mold easily due to its hydrophobic nature after silanization. Over that PDMS layer, we punched holes at the ends of channels and firmly placed that layer over the substrate which makes this top layer a plasma mask. Several different gas ingredients such as helium, heliumoxygen mixture and helium-nitrogen mixture were used to generate plasma. The generated plasma was injected from one hole which reached the other sides of the channels through the narrow openings, thus, exposing the substrate in a patterned way. We used two types of patterns; first type of pattern has two circular plasma inlets with $1 \mathrm{~mm}$ diameter, and a $75 \mu \mathrm{m}$ wide and $1 \mathrm{~cm}$ long straight channel between the inlets as shown in Fig 1(b). Liquid filament over this channel can be exploited to achieve waveguiding with air as the cladding medium. The second one has the same straight filament along with a disk with $250 \mu \mathrm{m}$ diameter located in the middle of the filament with a $70 \mu \mathrm{m}$ junction. This design is inspired by the coupled resonator optical waveguiding (CROW) phenomenon in which light passing through the waveguide is coupled to the disc to produce unique dispersion characteristics at the output port [38]. Subsequently, the substrate is kept in an oven at $100^{\circ} \mathrm{C}$ for $30 \mathrm{~min}$ to recover hydrophobicity. The same procedure can be repeated to obtain new pattern. The schematic diagram of the plasma exposure experimental setup is shown in Fig. 1.

For droplet experiments, substrate surface similar to the one used in the previous part was exposed to $f s$ laser to remove PDMS coating. The laser system used in experiments (Spectra Physics; Spitfire ${ }^{\circledR}$ Ace ${ }^{\mathrm{TM}}$ ) generated $120 \mathrm{fs}$ pulses at a wavelength of $800 \mathrm{~nm}$, pulse energy of $3.5 \mu \mathrm{J}$ and pulse repetition rate of $1 \mathrm{kHz}$. Schematic diagram of the laser micromachining system is shown in Fig. 2. Here, neutral density (ND) filter controls the power of the laser which must be critically controlled to obtain threshold value that allows PDMS ablation without damaging the glass slide. We used a $50 \mathrm{~mm}$ plano-convex spherical lens to focus the beam to the surface. Here, we placed the sample on a three-axis micromachining system which 

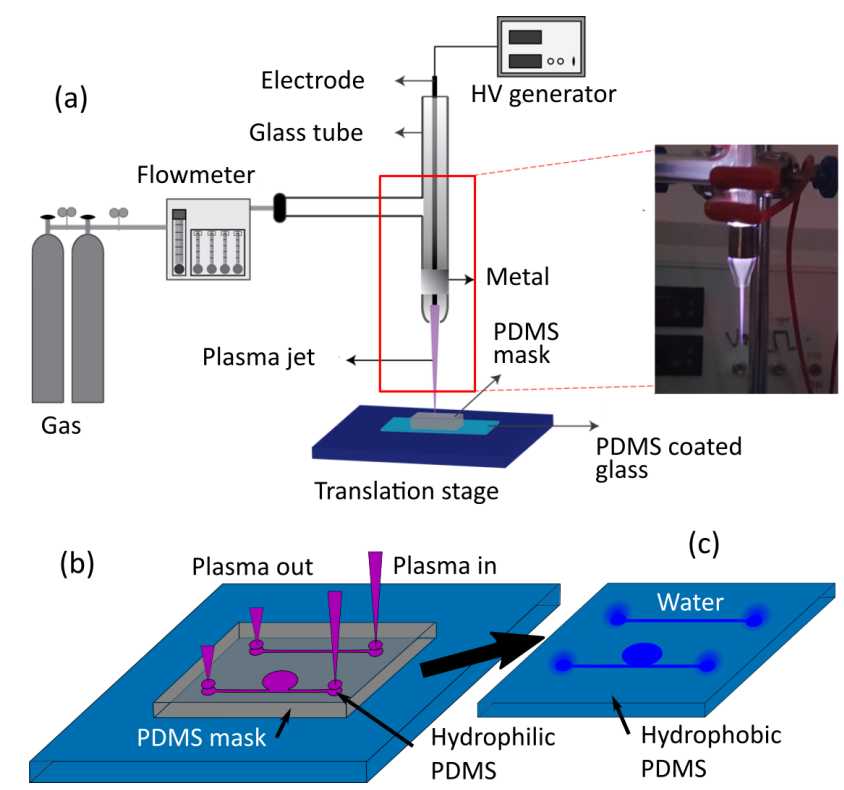

Figure 1 (a) Experimental setup for generating cold He plasma. (b) Selective plasma exposure through PDMS channels. (c) Water filaments over the substrate after removing the mask.

translates the sample with defined scan speed. During the laser patterning we monitored the surface of the sample in real-time using a CCD camera. The resultant hydrophilic track consisted of an inclined part at an angle of $10^{\circ}$ and a straight part with respect to the Hele-Shaw channel [see Fig. 4(a)]. Defined tracks have approximately $110 \mu \mathrm{m}$ width and $1 \mu \mathrm{m}$ depth. The top layer of PDMS was produced by soft lithography of the silicon mold which was obtained using $U V$ lithography technique with channels to generate microdroplets immersed in oil. Both, top layer and the substrate with ablated track were exposed to oxygen plasma at $1 \mathrm{~W}$ power for $45 \mathrm{sec}$ and bonded together permanently.

Light activated switchable surfaces were obtained using azobenzene and its derivative as an organic photochromic molecule. In order to coat the glass substrate with a layer prone to reversible isomerization under the $U V / v i s$ irradiation, we used 4-aminoazobenzene (4AAB) and 3-(triethoxysilyl)propyl isocyanate (TEPI) (SigmaAldrich) with the molecular weight of $197.24 \mathrm{~g} / \mathrm{mol}$ and $247.36 \mathrm{~g} / \mathrm{mol}$, respectively. We separately prepared $0.1 \mathrm{M}$ solutions of $4 \mathrm{AAB}$ and TEPI. We mixed these two solutions drop by drop at room temperature using a magnetic stirrer to react the amine groups on $4 \mathrm{AAB}$ with the isocyanate groups on TEPI where we obtained urea adduct of azobenzyene and TEPI. Subsequently, we dipped the glass slides into this reaction mixture for $20 \mathrm{sec}$, took out and dried at room temperature. This process was repeated 5 times

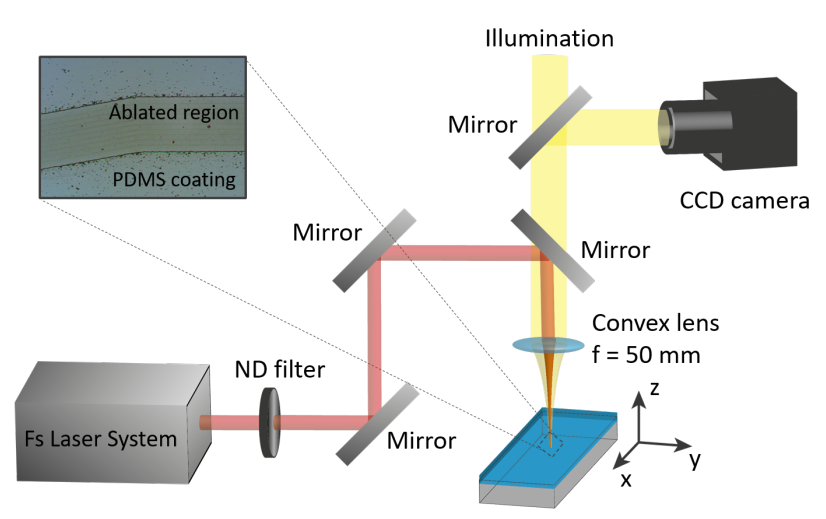

PDMS coated glass

Figure 2 Schematic diagram of laser ablation experimental setup. Inset shows the optical microscope image of the ablated track.

to obtain a fairly uniform coating of azobenzene-silane adduct in the trans isomer state onto the glass slide.

\section{Results and Discussion}

\subsection{Hydrophilic Patterning}

As a result of $\mathrm{He}$ plasma exposure to PDMS surface, the exposed region becomes hydrophilic. The hydrophilicity of the surface is determined by measuring the CA of a $10 \mu \mathrm{L}$ water droplet placed on the surface using a micro pipette. The snapshots of droplets before and after plasma exposure are shown in Fig. 3(a) and (b). The measured CAs of the droplets are $109^{\circ}$ and $8^{\circ}$ before and after plasma treatment, respectively. In order to obtain hydrophilic channels, PDMS microfluidic device with the patterns and inlet holes was firmly placed over the PDMS coated substrate in the hydrophobic state as described in the previous section. Plasma was injected from one hole which came out of the other hole after passing and activating the narrow opening over the substrate as shown in Fig. 1(b). Subsequently, the mask was removed from the substrate and a drop of water was put over one side which traveled to the other side through the hydrophilic channel. The microscope images of water filaments over the disc and the straight channel are shown in Fig. 3(c) and (d), respectively.

\subsection{Droplet Sorting}

The schematic diagram of the droplet sorting microfluidic device is shown in Fig. 4(a). The top layer of the device has two T-junctions for simultaneous droplet genera- 

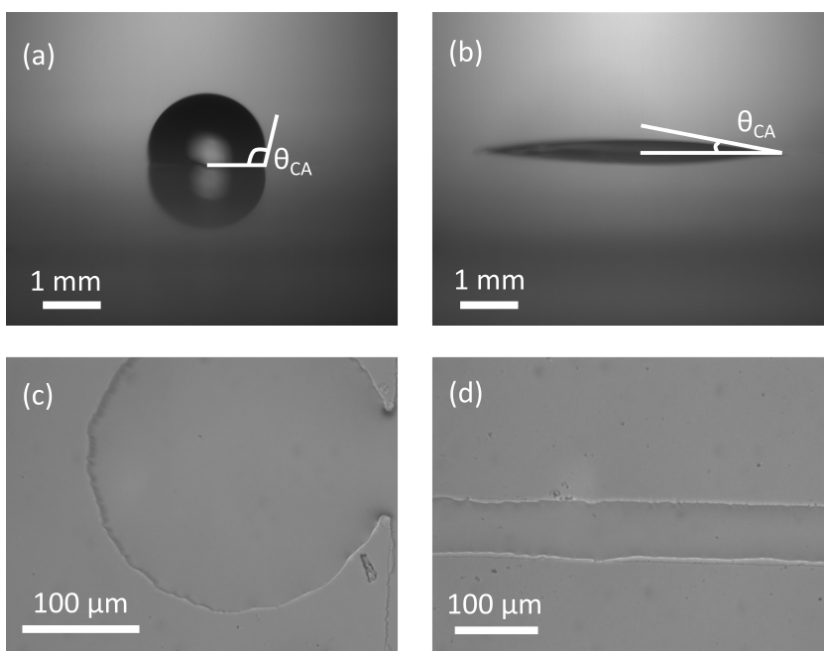

Figure 3 Water droplet (a) before plasma treatment $\left(C A=109^{\circ}\right)$ and (b) after plasma treatment $\left(C A=8^{\circ}\right)$. Optical microscope images of the water filaments over (c) disc and (d) straight hydrophilic patterns.

tion of two different liquids which enter the common HeleShaw channel hosting ablated track. Two liquids namely pure water and water/SDS (water mixed with sodium dodecyl sulphate (SDS) surfactant at $10 \mathrm{mM}$ concentration) are used as droplet liquids which are injected from top and bottom inlets respectively. Water/SDS droplets have low interfacial tension (IFT) so the capillary number $\left(C a=\eta U_{d} / \gamma\right)$ will be high as compared to the pure water droplets, where $\eta$ is the viscosity of the host fluid and $\gamma$ is the IFT between host liquid and the droplet. The size of the droplet is inversely proportional to $\mathrm{C} a$ as demonstrated by Leshansky et al., thus, water/SDS droplets are expected to have smaller size as compared to water droplets considering an identical flow rate and device geometry [39]. In order to reduce water droplet size at a given flow speed of the continuous phase, the inlet sizes in top T-junction are kept half of those of lower ones [40]. As a result, the droplets of both liquids are observed to be of similar sizes as shown in Fig. 4(b). Olive oil is injected in both continuous phase inlets at a flow rate of $25 \mu \mathrm{L} / \mathrm{h}$ and water or water/SDS is injected in the dispersed phase inlets at a flow rate of $1 \mu \mathrm{L} / \mathrm{h}$. This results in the generation of droplets of $100 \mu \mathrm{m}$ in diameter. In order to differentiate water from water/SDS droplets, we added blue dye into water.

In the absence of a droplet guiding track, the droplet motion is determined by the interplay between the fluid drag force $\left(\mathbf{F}_{D}\right)$, and friction force $\left(\mathbf{F}_{f}\right)$. $\mathbf{F}_{D}$ is obtained as the sum of pressure drag, $\mathbf{F}_{D, p}$, and viscous drag, $\mathbf{F}_{D, v}$, and is determined by the viscosity of the host fluid, geometrical parameters of the droplet and microfluidic channel, as well as average oil and droplet velocities [41, 42]. $\mathbf{F}_{f}$ acts on the moving droplet due to the channel walls and is mod-
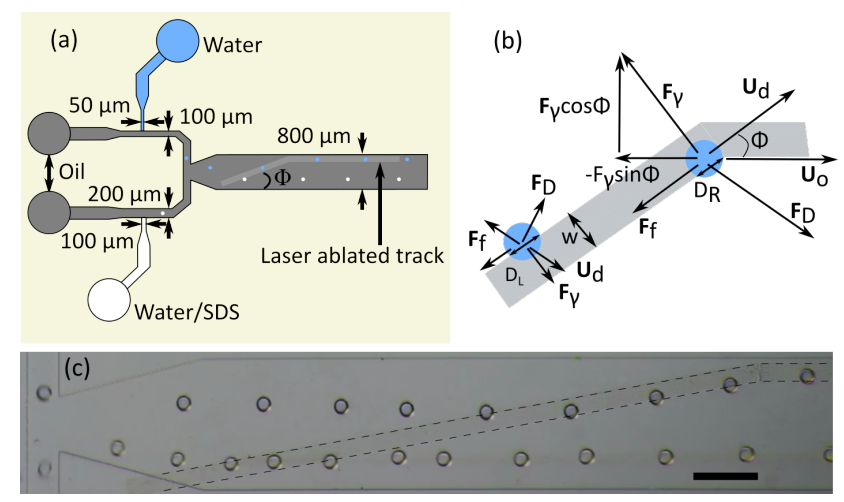

Figure 4 (a) Schematic diagram of the droplet sorting device. (b) Direction of forces acting on a droplet during its guidance. (c) Experimental results of droplet sorting. Scale bar is $400 \mu \mathrm{m}$.

eled as $\mathbf{F}_{f}=-\beta \mathbf{U}_{d}$ where $\beta$ is the friction coefficient [42]. When the droplet enters the guiding track, a third force, namely, confinement force $\left(\mathbf{F}_{\gamma}\right)$ also acts on the droplet which forces the droplet to move in the inclined direction. The directions of all forces acting on the droplet when it touches left or right track edge are shown in Fig. 4(b). $\mathbf{F}_{\gamma}$ is composed of two forces, one is due to the droplet surface energy gradient effect caused by $\sim 1 \mu$ m deep topographic feature [41] and the other is due to the wetting contrast between the track and surrounding region [43]. Wetting contrast causes the droplet to stay inside the track due to its more hydrophilic nature. The total confinement force is approximated as:

$$
F_{\gamma}=-\gamma \frac{\Delta A}{2 R}+\gamma\left(D_{R}-D_{L}\right)\left(\cos \theta_{\text {in }}-\cos \theta_{\text {out }}\right),
$$

Where $\gamma$ is the IFT between oil and droplet, $\theta_{\text {in }}$ and $\theta_{\text {out }}$ are the CAs of droplets inside and outside the guiding track, and $D_{L}$ and $D_{R}$ are the intersection lengths of droplets with the left and right track edges, respectively [Fig. 4(b)]. $\Delta A$ is the change in the droplet surface area when it enters from outside to the inside of the track and $R$ is its radius. Fundamentally, there are no inertial forces acting on the moving droplets at low Reynold number limit and the algebraic sum of all forces acting on the droplet is always equal to 0, i.e. $\mathbf{F}_{D}+\mathbf{F}_{f}+\mathbf{F}_{\gamma}=0$. Knowing the direction of $\mathbf{F}_{\gamma}$ (perpendicular to the edges) and $\mathbf{F}_{f}$ (opposite to the droplet motion), $\mathbf{F}_{D}$ is evaluated accordingly as a consequence to the equilibrium condition. The IFTs of water and water/SDS with olive oil are $\gamma_{w}=16 \mathrm{mN} / \mathrm{m}$ and $\gamma_{w / s d s}=2.5 \mathrm{mN} / \mathrm{m}$ which implies that water/SDS droplets experience almost an order of magnitude smaller confinement force at the given flow conditions [34]. That is why, water/SDS droplets are observed to move straight without following the track, whereas, water droplets are trapped inside the track and follow it as shown in Fig. 
4(c). The CAs of water (water/SDS) are measured to be $172^{\circ}\left(177^{\circ}\right)$ and $167.5^{\circ}\left(173^{\circ}\right)$ over PDMS and ablated regions, respectively. The low CA of water and water/SDS droplets over track surface is attributed to the hydrophilic nature of uncovered glass, however, the hydrophilicity is not too impressive due to the presence of traces of residual PDMS after laser ablation. The detailed topographic examination of track and PDMS surface can be found in the ESI of our previous work which clearly shows the inhomogeneities over the track surface [34]. The experimental results of sorting of two droplets are shown in Fig. 4(c). Water droplets experience much higher $F_{\gamma}$ due to higher IFT, so they are guided along the track. Water/SDS droplets barely get deflected along the track since they experience extremely low $F_{\gamma}$.

\subsection{Photoisomerization of Azobenzene}

Fig. 5 shows the absorption spectra of $4 \mathrm{AAB}$ coated glass substrate between $300-600 \mathrm{~nm}$ wavelength range. We used widely tunable Ti:Sapphire femtosecond laser (Coherent, Chameleon) to irradiate the substrates. The numbers on the lines indicate the order of $U V / v i s$ exposure. Spectrum (1) that is obtained following exposure of the surface to $370 \mathrm{~nm} U V$ light for 5 min corresponds to cis conformation where $4 \mathrm{AAB}$ exhibits absorption maxima at around $340 \mathrm{~nm}$ and $440 \mathrm{~nm}$. When the surface is exposed to $440 \mathrm{~nm}$ visible light for $15 \mathrm{~min}$, cis to trans transition occurs and absorption peak at $U V$ is shifted to $370 \mathrm{~nm}$, as seen in Spectrum (2). When this cycle is repeated, cis to trans isomerization can be obtained in a fully reversible manner as shown in Spectra (3) and (4).

We also studied reversible wettability of $4 \mathrm{AAB}$ coated surfaces under $U V / v i s$ exposure by water CA measurements. It is known that azobenzene-modified coatings on flat surfaces exhibit only minor changes (less than $10^{\circ}$ ) in the CA [44]. Accordingly, we measured the CAs as $62.9^{\circ}$ and $58.2^{\circ}$ over the $4 \mathrm{AAB}$ coated surface in trans-state and cis-state, respectively. Although the CA change is very small for our photoswitchable surface, we observed reversible wettability transitions for several cycles as shown in Fig. 5(b). In order to enhance CA difference under UV/vis irradiation, surfaces with fluorinated azobenzene films combined with rough substrates can be produced [24]. With such surfaces, transitions between superhydrophobicity and superhydrophilicity have been reported.

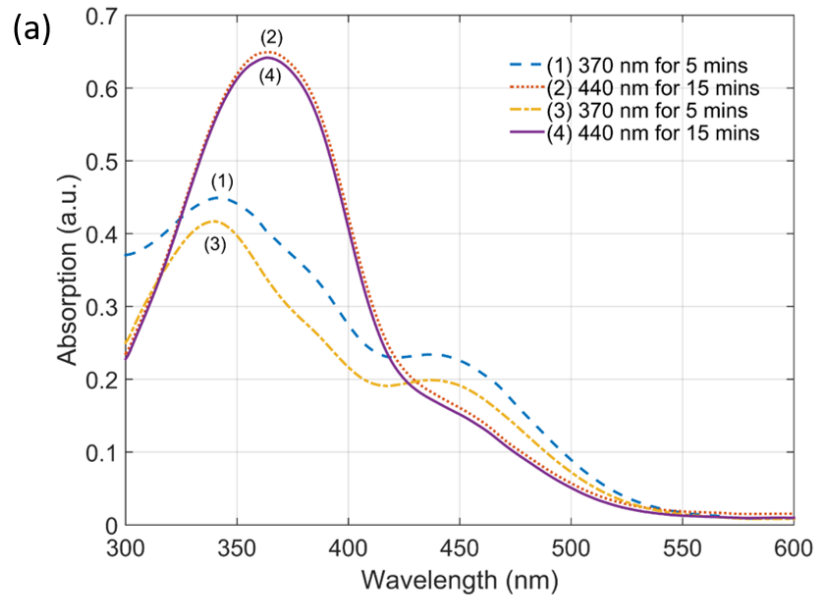

(b)

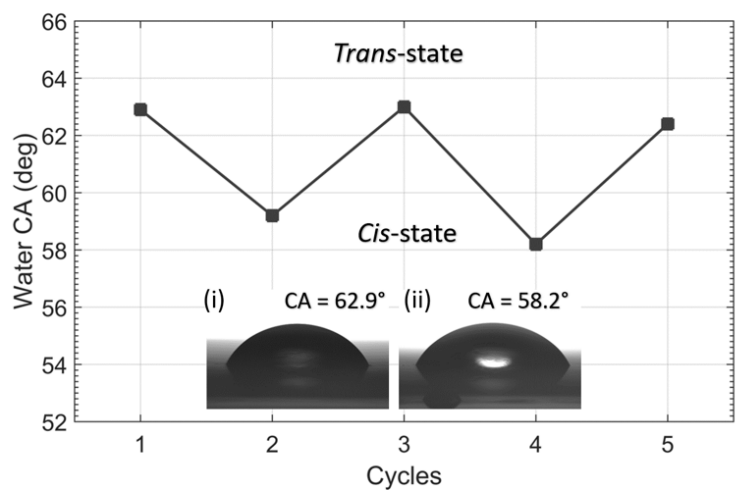

Figure 5 (a) Absorption spectrum of 4-Aminoazobenzene coated glass surface after (1),(3) $U V$ irradiation for $5 \mathrm{~min}$ (cis-state) and (2),(4) after vis irradiation for $15 \mathrm{~min}$ (trans-state). Numbers indicate the order of cis-to-trans conversion, respectively. (b) Reversible wettability transitions with UV/vis irradiation. Inset shows the water droplet profiles on (i) vis irradiated and (ii) UV irradiated surfaces dueing cycles 1 and 4 , respectively. The water CA is $62.9^{\circ}$ and $58.2^{\circ}$ in trans-state and cis-state, respectively.

\section{Conclusions}

In this paper, we explored unique aspects of PDMS for developing smart surfaces to obtain high resolution wettable channels for fluid dynamics and mass transport. In addition to its elementary use for fabrication of microfluidic devices, we have reported simple ways to modify surface properties of PDMS both chemically and physically. Two important applications of manipulating its wetting properties are experimentally demonstrated; reconfigurable open surface patterning and passive droplet separation with a high success rate.

The plasma induced switching of extreme regimes of wettability produces extremely narrow $(\sim 70 \mu \mathrm{m})$ and tightly confined liquid filaments which possess high surface to volume ratio leading to quick and efficient analyte absorption at high concentration and detection sen- 
sitivity. The chemical detection can be carried out in vapor phase compatible with ambient humidity which is routinely available at the airports and public venues [33]. Reconfigurability achieved in a quick span adds to the ease and flexibility, thus, allowing fabrication of complex networks which are impossible to achieve with irreversible machining methods. Reconfigurable free surface patterns are also finding applications in optofluidics such as filtering, optical resonance, biosensing and biolasing due to the smooth interface, easy replacement and wide range of refractive indices of fluids [45].

Another possibility of modifying the PDMS surface is to expose it with high intensity laser pulses which remove thin coating and uncover hydrophilic glass substrate. Although, the hydrophilicity achieved in this way is not very significant, yet, it is good enough for achieving manipulation of water droplets immersed in oil. The passive separation of droplet assays demands accurate and optimized channel design and orientation to achieve high throughput screening at a rapid speed. By building such optimized and well-oriented hydrophilic tracks, we obtained sorting of water and water/SDS droplets in the same chip due to different IFT and $F_{\gamma}$. The proposed scheme is highly economical, passive, autonomous and trivial incorporating only the different physics of droplets and device geometry.

Light activated surface modification is expected to open new prospects in closed microfluidic systems to externally define submicron tracks within microfluidic channels which can last for longer periods of time before hydrophobic recovery. With the use of photo mask, this scheme provides better control with extremely high resolution required specifically in nanofluidics and photonics for single mode waveguide operation. Azobenzene and many of its derivatives offer appreciable changes in dipole moment and wettability upon exposure to $U V / v i s$ radiation which can be recovered substantially over several cycles. As a result, water CA changes solely due to chemical activity without altering the topography of the coated layer. We experimentally investigated the conversion of $4 \mathrm{AAB}$ from cis to trans (trans to cis) upon exposure to light at $\lambda=440 \mathrm{~nm}(\lambda=370 \mathrm{~nm})$.

This work was supported by TÜBİTAK (Grant No. 117F348) and HEC Pakistan (Ph.D. scholarship of Z.R.). We thank M. Muradoglu for fluid dynamics modeling, and A. Jonáš and A. Erten for useful discussions. We also thank KUYTAM (Koç University Surface Science and Technology Center) for laser ablation experiments and characterization measurements.

\section{References}

[1] Iskender Yilgor, Sevilay Bilgin, Mehmet Isik, and Emel Yilgor. Facile preparation of superhydrophobic polymer surfaces. Polymer, 53(6):1180-1188, 2012.

[2] Robert J. Daniello, Nicholas E. Waterhouse, and Jonathan P. Rothstein. Drag reduction in turbulent flows over superhydrophobic surfaces. Phys. Fluids, 21(8):085103, 2009.

[3] Adel M.A. Mohamed, Aboubakr M.Abdullah, and Nathalie A.Younan. Corrosion behavior of superhydrophobic surfaces: A review. Arab. J. Chem., 8(6):749-765, 2015.

[4] Almeida Riberet. Anti-fog coatings using the super-hydrophobic approach, 2008.

[5] Ludmila B. Boinovich and Alexandre M. Emelyanenko. Anti-icing potential of superhydrophobic coatings. Mendeleev Commun., 23(1):3-10, 2013.

[6] Alexandr Jonas, Mehdi Aas, Yasin Karadag, Selen Manioglu, Suman Anand, David McGloin, Halil Bayraktar, and Alper Kiraz. In vitro and in vivo biolasing of fluorescent proteins suspended in liquid microdroplet cavities. Lab Chip, 14(16):3093-3100, 2014.

[7] Jaesung Son, Shreya Kundu, Lalit K. Verma, Mridul Sakhuja, Aaron J. Danner, Charanjit S. Bhatia, and Hyunsoo Yang. A practical superhydrophilic self cleaning and antireflective surface for outdoor photovoltaic applications. J. Adhes. Sci. Technol., 98:46-51, 2012.

[8] Liang Zhang, Ning Zhao, and Jian Xu. Fabrication and application of superhydrophilic surfaces: a review. J. Adhes. Sci. Technol., 28(9):769-790, 2014.

[9] Y. Takata, S. Hidaka, M. Masuda, and T. Ito. Pool boiling on a superhydrophilic surface. Int. J. Energy Res., 27(11):1-9, 2003.

[10] Giampaolo Mistura and Matteo Pierno. Drop mobility on chemically heterogeneous and lubricant-impregnated surfaces. Adv. Phys. X, 2(3):591-607, 2017.

[11] Jana Krenkova and Frantisek Foret. Immobilized microfluidic enzymatic reactors. Electrophoresis, 25(21-22):3550-3563, 2004.

[12] Moo-Yeal Lee, Aravind Srinivasan, Bosung Ku, and Jonathan S. Dordick. Multienzyme catalysis in microfluidic biochips. Biotechnol. Bioeng, 83(1):20-28, 2003.

[13] Abdennour Abbas, Anthony Treizebre, Philippe Supiot, NourEddine Bourzgui, Didier Guillochon, Dominique VercaigneMarko, and Bertrand Bocquet. Cold plasma functionalized terahertz biomems for enzyme reaction analysis. Biosens. Bioelectron., 25(1):154-160, 2009.

[14] Kim Shyong Siow, Leanne Britcher, Sunil Kumar, and Hans J. Griesser. Plasma methods for the generation of chemically reactive surfaces for biomolecule immobilization and cell colonization - a review. Plasma Process Polym., 3(6):392-418, 2006.

[15] Fevzihan Basarir, Nguyen Cuong, Woo-Keun Song, and Tae-Ho Yoon. Surface modification via plasma polymerization of allylamine for antibody immobilization. Macromol. Symp., 249250(1):61-66, 2007.

[16] Natalie Wagner and Patrick Theato. Light-induced wettability changes on polymer surfaces. Polymer, 55:3436-3453, 2014.

[17] Dieter't Mannetje, Somnath Ghosh, Rudy Lagraauw, Simon Otten, Arjen Pit, Christian Berendsen, Jos Zeegers, Dirk van den Ende, and Frieder Mugele. Trapping of drops by wetting defects. Nature Comm., 5(3559):1-7, 2014. 
[18] Carlo Rigoni, Matteo Pierno, Giampaolo Mistura, Delphine Talbot, Rene Massart, Jean-Claude Bacri, and Ali AbouHassan. Static magnetowetting of ferrofluid drops. Langmuir, 32(30):7639-7646, 2016.

[19] Kiyomi Matsuda, Hirotaka Yamamoto, Ayumi Kashiwada, Kazunori Yamada, and Mitsuo Hirata. Surface hydrophilization of ptfe plates by oxygen plasma pre-treatment and photografting - dependence on solvent composition of monomer solution. J. Photopolym. Sci. Technol., 18(2):257-262, 2005.

[20] P. K. Chu, J. Y. Chen, Langping Wang, and Lily Huang. Plasmasurface modification of biomaterials. Electrophoresis, 36(56):143-206, 2002.

[21] H. Hillborg and U. W. Gedde. Hydrophobicity recovery of polydimethylsiloxane after exposure to corona discharges. Polymer, 39(10):1991-1998, 1998.

[22] Joong Tark Han, Sangcheol Kim, and Alamgir Karim. Uvo-tunable superhydrophobic to superhydrophilic wetting transition on biomimetic nanostructured surfaces. Langmuir, 23(5):26082614, 2007. PMID: 17269808.

[23] Gerald Blanco-Gomez, Leonard M. Flendrig, and Jonathan M. Cooper. Hysteresis and reversibility of a superhydrophobic photopatternable silicone elastomer. Langmuir, 26(10):7248-7253, 2010.

[24] Ho Sun Lim, Joong Tark Han, Donghoon Kwak, Meihua Jin, and Kilwon Cho. Photoreversibly switchable superhydrophobic surface with erasable and rewritable pattern. J. Am. Chem. Soc., 128(45):14458-14459, 2006.

[25] Wei Sun, Shuxue Zhou, Bo You, and Limin Wu. A facile method for the fabrication of superhydrophobic films with multiresponsive and reversibly tunable wettability. J. Mat. Chem. A, 1(9):3146-3154, 2013.

[26] Gwendoline Petroffe, Chao Wang, Xavier Sallenave, Gjergji Sini, Fabrice Goubard, and Sebastien Peralta. Fast and reversible photo-responsive wettability on $\mathrm{tio}_{2}$ based hybrid surfaces. J. Mat. Chem. A, 3(21):11533-11542, 2015.

[27] Emel Yilgor, Orkun Kaymakci, Mehmet Isik, Sevilay Bilgin, and Iskender Yilgor. Effect of uv/ozone irradiation on the surface properties of electrospun webs and films prepared from polydimethylsiloxane-urea copolymers. Appl. Surf. Sci., 258(10):4246 - 4253, 2012

[28] Sevilay Bilgin, Mehmet Isik, Emel Yilgor, and Iskender Yilgor. Hydrophilization of silicone-urea copolymer surfaces by uv/ozone: Influence of pdms molecular weight on surface oxidation and hydrophobic recovery. Polymer, 54(25):6665-6675, 2013.

[29] Zeeshan Rashid, Ipek Atay, Seren Soydan, M. Baris Yagci, Alexandr Jonas, Emel Yilgor, Alper Kiraz, and Iskender Yilgor. Reversible switching of wetting properties and erasable patterning of polymer surfaces using plasma oxidation and thermal treatment. Appl. Surf. Sci., 441(28):841-852, 2018.

[30] Jennifer L. Fritz and Michael J. Owen. Hydrophobic recovery of plasma-treated polydimethylsiloxane. J. Adhes., 54(1-2):33-45, 1995.

[31] Lingfei Hong and Tingrui Pan. Surface microfluidics fabricated by photopatternable superhydrophobic nanocomposite. Microfluid Nanofluid, 10(5):991-997, 2011.

[32] Siyuan Xing, Ryan S. Harake, and Tingrui Pan. Droplet-driven transports on superhydrophobic-patterned surface microfluidics. Lab Chip, 11(21):3642-3648, 2011.
[33] Brian D. Piorek, Seung Joon Lee, Martin Moskovits, and Carl D. Meinhart. Free-surface microfluidics/surface-enhanced raman spectroscopy for real-time trace vapor detection of explosives. Anal. Chem., 84(22):9700-9705, 2012.

[34] Zeeshan Rashid, Umut Can Coskun, Yagiz Morova, Berna Morova, Asuman Asikoglu Bozkurt, Ahmet Erten, Alexandr Jonas, Selcuk Akturk, and Alper Kiraz. Guiding of emulsion droplets in microfluidic chips along shallow tracks defined by laser ablation. Microfluid Nanofluid, 11(160):1-12, 2017.

[35] Henrik Hillborg, Nikodem Tomczak, Attila Olah, Holger Schonherr, and G. Julius Vancso. Nanoscale hydrophobic recovery: A chemical force microscopy study of uv/ozone-treated crosslinked poly(dimethylsiloxane). Langmuir, 20(3):785-794, 2003.

[36] Shazia Bashir, Muhammad Bashir, Xavier Casadevall i Solvas, Julia M. Rees, and William B. Zimmerman. Hydrophilic surface modification of pdms microchannel for $\mathrm{o} / \mathrm{w}$ and $\mathrm{w} / \mathrm{o} / \mathrm{w}$ emulsions. Micromachines, 6(10):1445-1458, 2015.

[37] H. Hillborg, J.F. Ankner, U.W. Gedde, G.D. Smith, H.K. Yasuda, and K. Wikstrom. Crosslinked polydimethylsiloxane exposed to oxygen plasma studied by neutron reflectometry and other surface specific techniques. Polymer, 41(3):6851-6863, 2000.

[38] Jacob Scheuer, George T. Paloczi, Joyce K. S. Poon, and Amnon Yariv. Coupled resonator optical waveguides: Toward the slowing and storage of light. Opt. Photonics News, 16(2):36-40, 2005.

[39] Alexander M. Leshansky and Len M. Pismen. Breakup of drops in a microfluidic t junction. Phys. Fluids, 21(2):023303, 2009.

[40] Piotr Garstecki, Michael J. Fuerstman, Howard A. Stonec, and George M. Whitesides. Formation of droplets and bubbles in a microfluidic t-junction-scaling and mechanism of break-up. Lab Chip, 6(3):437-446, 2006.

[41] Remi Dangla, Sungyon Lee, and Charles N. Baroud. Trapping microfluidic drops in wells of surface energy. Phys. Rev. Lett., 107(12):12450, September 2011.

[42] Tsevi Beatus, Roy H. Bar-Ziv, and Tsvi Tlusty. The physics of 2d microfluidic droplet ensembles. Phys. Rep., 516(3):103-145, March 2012.

[43] Arjen M. Pit, Sander Bonestroo, Daniel Wijnperle, Michel H. G. Duits, and Frieder Mugele. Electrode-assisted trapping and release of droplets on hydrophilic patches in a hydrophobic microchannel. Microfluid Nanofluid, 20(123):1-12, 2016.

[44] Kunihiro Ichimura, Sang-Keun Oh, and Masaru Nakagawa. Light-driven motion of liquids on a photoresponsive surface. Science, 288(5471):1624-1626, 2000.

[45] Alexandr Jonas, Berna Yalizay, Selcuk Akturk, and Alper Kiraz. Free-standing optofluidic waveguides formed on patterned superhydrophobic surfaces. Appl. Phys. Lett., 104(9):091123, 2014. 